\title{
Interaction of glycophorin A with lectins as measured by surface plasmon resonance (SPR)
}

\author{
Bożena Krotkiewska ${ }^{1}$, Marta Pasek $^{2}$ and Hubert Krotkiewski ${ }^{2 凶}$ \\ ${ }^{1}$ Department of Medical Biochemistry, University Medical School, Wroctaw, Poland; \\ ${ }^{2}$ Department of Immunochemistry, Ludwik Hirszfeld Institute of Immunology and Experimental \\ Therapy, Polish Academy of Sciences, Wroctaw, Poland
}

Received: 07 February, 2002; revised: 07 May, 2002; accepted: 21 May, 2002

Key words: biosensor, surface plasmon resonance, human glycophorin, lectins, glycoproteins

\begin{abstract}
Glycophorin A (GPA), the major sialoglycoprotein of the human erythrocyte membrane, was isolated from erythrocytes of healthy individuals of blood groups A, B and $O$ using phenol-water extraction of erythrocyte membranes. Interaction of individual GPA samples with three lectins (Psathyrella velutina lectin, PVL; Triticum vulgaris lectin, WGA and Sambucus nigra I agglutinin SNA-I) was analyzed using a BIAcore ${ }^{\mathrm{TM}}$ biosensor equipped with a surface plasmon resonance (SPR) detector. The experiments showed no substantial differences in the interaction between native and desialylated GPA samples originating from erythrocytes of either blood group and each of the lectins. Desialylated samples reacted weaker than the native ones with all three lectins. PVL reacted about 50-fold more strongly than WGA which, similar to PVL, recognizes GlcNAc and Neu5Ac residues. SNA-I lectin, recognizing $\alpha 2-6$ linked Neu5Ac residues, showed relatively weak reaction with native and only residual reaction with desialylated GPA samples. The data obtained show that SPR is a valuable method to determine interaction of glycoproteins with lectins, which potentially can be used to detect differences in the carbohydrate moiety of individual glycoprotein samples.
\end{abstract}

The BIAcore ${ }^{\mathrm{TM}}$ instrument is a biosensor designed to investigate interactions between different molecules (biospecific interaction ana- lysis); the instrument makes it possible to analyze these interactions in real time. The study of biomolecular interactions is of basic impor-

\footnotetext{
${ }^{{ }^{*}}$ This work was supported by the University Medical School in Wrocław, grant no. 312

${ }^{\bowtie}$ Corresponding author: Department of Immunochemistry, Ludwik Hirszfeld Institute of Immunology and Experimental Therapy, Polish Academy of Sciences, R. Weigla 12, 53-114 Wrocław, Poland; tel. (48 71) 373 2274, fax (48 71) 373 2587; e-mail: krotkiew@immuno.iitd.pan.wroc.pl

Abbreviations: GlcNAc, $N$-acetylglucosamine; GPA, human glycophorin A; GPA-A, GPA-B, GPA-O; glycophorin isolated from blood group A, B and $\mathrm{O}$ erythrocytes, respectively; Neu5Ac, $N$-acetylneuraminic acid; PVL, Psathyrella velutina lectin; RA, rheumatoid arthritis; RU, resonance units; SNA-I, Sambucus nigra I agglutinin; SPR, surface plasmon resonance; WGA, Triticum vulgaris agglutinin.
} 
tance in searching and understanding the processes of molecular recognition and biological function of molecules. The procedure of analysis is as follows: one of the analyzed substances, called the ligand, is immobilized in the working channel of a specially designed sensor chip; the other substance, called the analyte, is in a solution which, during the experiment, is in contact with the ligand immobilized on the sensor chip. This physical contact enables the interaction between the two analyzed substances to take place. BIAcore instrument has a flow cell to deliver a constant supply of the analyte to the sensor surface during the association phase; during the dissociation phase the flow system is essential for removing free analyte released from the working surface. The mode of detection, used in the BIAcore biosensor, is the surface plasmon resonance (SPR) technique. SPR is a phenomenon which occurs between incoming photons and the electrons on the surface of a thin gold film coated onto a glass support on the sensor chip. At a specific wavelength and a specific angle of incident light the energy is transferred to the electrons in the gold surface causing the reflected light to disappear. This angle of non-reflectance, called the resonance angle, changes in response to the change of the refractive index in the vicinity of the gold surface. A signal from the detector, measured in resonance units (RU), is generated when two interacting molecules form a complex. The signal is proportional to the change in mass (for proteins $1 \mathrm{RU}$ is equal to approx. $1 \mathrm{pg} / \mathrm{mm}^{2}$ ) (Stenberg et al., 1991). Using the BIAcore instrument interactions between various molecules can be measured, e.g., antigen and antibody, ligand and its receptor, enzyme and its substrate, lectin and glycoprotein. Therefore, one can study the binding of nucleic acids, lipid micelles, peptides, drugs and other small molecules. The technology is valuable in a wide range of applications, from basic research to drug discovery and development, monoclonal antibody characterization, infectious disease research, etc.
The BIAcore system measures the change in surface concentration of the analyzed substance down to $1 \mathrm{pg} / \mathrm{mm}^{2}$ and can monitor these changes on a time scale of $0.1 \mathrm{~s}$. Moreover, the measurements allow, for typical molecular binding, accurate kinetic calculations in the range of $10^{3}-10^{7} \mathrm{M}^{-1} \mathrm{~s}^{-1}$ for association rate constant $\left(k_{\mathrm{a}}\right)$ and $10^{-6}-10^{-1} \mathrm{~s}^{-1}$ for dissociation rate constant $\left(k_{\mathrm{d}}\right)$, together with concomitant association constant $K_{\mathrm{A}}$ $\left(10^{4}-10^{12} \mathrm{M}^{-1}\right)$ and dissociation constant $K_{\mathrm{D}}$ $\left(10^{-4}-10^{-12} \mathrm{M}\right)$. An important advantage of this method is the lack of labeling requirements, which means simplicity and uniformity of different applications. The use of the BIAcore biosensor in biochemical and biomedical sciences is growing extensively (Rich \& Myszka, 2000). Not only soluble substances can be analyzed using this methodology, but also larger particles like whole cells, e.g. erythrocytes (Quinn et al., 1997).

In this work we applied a BIAcore-1000 biosensor to investigate the interaction of glycophorin A (GPA), native and desialylated, with three different lectins. Glycophorin A is the major sialoglycoprotein of the human erythrocyte membrane (Lisowska, 2001). This glycoprotein contains two types of glycans, i.e. one $\mathrm{N}$-glycan and about $15 \mathrm{O}$-glycans per molecule; it has been used in numerous immunochemical and biochemical structural investigations, e.g. (Krotkiewski et al., 1993; 1997; 1999; Podbielska \& Krotkiewski, 2000). GPA contains bisecting $N$-acetylglucosamine in the N-linked glycan (Yoshima et al., 1980). Most of the O-glycans of GPA contain two Neu5Ac residues (Lisowska et al., 1980). Together with two sialic acid residues from the N-glycan they represent receptors, which can be recognized by appropriate lectin. The analyses performed in this work regarded glycophorin samples derived from individual human donors of defined $\mathrm{ABO}$ blood group. We used the following lectins: Psathyrella velutina lectin (PVL), Triticum vulgaris lectin (wheat germ agglutinin, WGA) and Sambucus nigra lectin (SNA-I). The first two exhibit the same specificity, i.e. 
they recognize GlcNAc and Neu5Ac residues. The third lectin reacts with sialic acid bound $\alpha 2-6$ to $\mathrm{Gal} / \mathrm{GalNAc}$ which means it is sensitive to desialylation of the reactant. The aim of this study was to establish immobilization and regeneration procedures, sensitivity of GPA interaction with the chosen lectins, influence, if any, of the blood group on GPA-lectin interaction and to check the reproducibility of the results.

\section{MATERIALS AND METHODS}

Materials. Psathyrella velutina lectin (PVL) was from WAKO (Germany), Triticum vulgaris lectin (WGA) from Pharmacia (Sweden) and Sambucus nigra lectin (SNA-I) from Sigma (U.S.A.). BIAcore sensor chips CM5 were from Biacore AB (Uppsala, Sweden). Two chemicals used for the sensor chip activation procedure: EDC ( $N$-ethyl- $N{ }^{\prime}$-(3-dimethylaminopropyl)carbodiimide hydrochloride) and NHS ( $N$-hydroxysuccinimide) were from Sigma (U.S.A.); ethanolamine was from Merck (Germany). All solutions were prepared using high quality water from a Milli Q apparatus (Millipore), working in a reversed-osmose mode (electrical resistance $\geq$ $16 \mathrm{M} \Omega$ ). The binding buffer (HBS, $10 \mathrm{mM}$ Hepes/NaOH, pH 7.4, 0.15 M NaCl, $3.4 \mathrm{mM}$ EDTA, 0.05\% Tween 20) was prepared according to the manufacturer's prescription.

Instrumentation. To measure interactions between glycophorin and the lectins a BIAcore-1000 instrument was used (Pharmacia Biosensor AB, Uppsala, Sweden). The technological fluctuations of the base line were \pm 5 resonance units (RU). The sensor chips CM5 with carboxymethylated dextran matrix were used throughout all experiments. The kinetic data from individual binding experiments were determined using the BIAevaluation software (Pharmacia, Sweden). Instrument compartment with the sensor chip was thermostated at $20^{\circ} \mathrm{C}$ during all experiments. All the solutions were filtered
(0.22 $\mu \mathrm{m}$, Millipore) and degassed before use.

Human glycophorin A. Glycophorin was isolated from the membranes of outdated human erythrocytes of individual subjects with blood groups A, B and O by phenol-water extraction, as described previously (Lisowska et $a l ., 1987)$. The yield of crude GPA preparation, obtained from one individual blood sample $(300 \mathrm{ml})$, was about 50-60 mg. For comparative purposes three crude glycophorin preparations: GPA-A, GPA-B and GPA-O were purified by gel filtration on a Sephadex G-200 column in a buffer containing 1\% SDS (Podbielska \& Krotkiewski, 2000). After removing the bulk of SDS from the purified samples by dialysis against $50 \%$ ethanol and then water the samples were treated with Bio-Beads SM-2 adsorbent (BioRad) to remove residual detergent. The content of SDS in the final purified GPA samples was determined using a colorimetric method (Hayashi, 1975). The recovery of glycophorin after purification was about $60 \%$ of the starting material.

Desialylation. Desialylation of glycophorin samples (1-2 mg) was performed in $0.05 \mathrm{M}$ sulfuric acid at $60^{\circ} \mathrm{C}$ for $4 \mathrm{~h}$. After neutralization with $\mathrm{NaOH}$ the samples were dialyzed against distilled water. The residual content of Neu5Ac in the desialylated GPA samples was determined by a colorimetric method (Jourdian et al., 1971). It was lower than 4.0\% of the protein content, which is in agreement with our previous experiments which showed that desialylated GPA never lacks sialic acid completely.

Protein determination. Protein was determined on a micro scale (96-well polystyrene plates) using a colorimetric method with bicinchoninic acid (BCA) (Smith et al., 1985). Bovine serum albumin (BSA) was used as a standard (Sigma, U.S.A.).

Immobilization of the ligand on the sensor surface. The immobilization was performed on CM5 sensor chips, according to the manufacturer's description based on published data (Johnsson et al., 1991). Briefly, the 
procedure was as follows: step no. 1 preconcentration. Aliquots of $0.01 \mathrm{M}$ sodium acetate buffer of different $\mathrm{pH}$ values $\mathrm{pH} 4.0$, $4.5,5.0,5.5,6.0$ ) were mixed with sample solutions, each containing lectin (5-20 $\mu \mathrm{g})$. These solutions were then used in binding experiments in 2-min pulses. The buffer, for which the binding was the highest was chosen for the immobilization procedure. Step no. $2-a c$ tivation of the carboxymethylated dextran surface. This was done using EDC/NHS system, i.e. $50 \mu \mathrm{l}$ of $0.04 \mathrm{M}$ NHS solution was mixed with $75 \mu \mathrm{l}$ of $0.16 \mathrm{M}$ EDC; the final solution was pumped across the working channel (5 $\mu \mathrm{l} / \mathrm{min}$ for $7 \mathrm{~min}$ ), followed by HBS buffer. After this step the baseline should rise by $c a$ 150-200 RU. Step no. 3 - ligand immobilization. Lectin sample (5-20 $\mu \mathrm{g})$ was dissolved in $0.01 \mathrm{M}$ sodium acetate buffer with the previously determined optimum $\mathrm{pH}$; this solution was pumped across the channel ( $5 \mu \mathrm{l} / \mathrm{min})$ in several pulses, together lasting from 2 to 7 $\mathrm{min}$. The rise of the baseline at this step of the procedure was between 2500-8000 RU. Step no. 4 -deactivation. The residual NHS esters, generated on the working channel surface, were destroyed with $1 \mathrm{M}$ ethanolamine, $\mathrm{pH}$ 8.5, for $12 \mathrm{~min}$. Step no. 5-washing. The final washing of the channel was performed using $0.5 \mathrm{M} \mathrm{NaCl}$ for $10 \mathrm{~min}$. Between each of the steps the working channel was washed with HBS buffer.

Binding studies. For the binding experiments the following durations, solution compositions and flow rates were used: $1^{\circ}$ for PVL and WGA lectin: initial HBS flow $-5 \mu \mathrm{l} / \mathrm{min}$, association $-2 \mu \mathrm{l} / \mathrm{min}$ for $10 \mathrm{~min}$, dissociation $-20 \mu \mathrm{l} / \mathrm{min}$ of HBS for $3 \mathrm{~min}$, regeneration $-20 \mu \mathrm{l} / \mathrm{min}$ of $0.2 \mathrm{M} \mathrm{GlcNAc}$ in HBS for $10 \mathrm{~min}$, final HBS flow $-20 \mu \mathrm{l} / \mathrm{min}$ for $5 \mathrm{~min}$, $2^{\circ}$ for SNA lectin: initial HBS flow $-5 \mu \mathrm{l} / \mathrm{min}$, association $-2 \mu \mathrm{l} / \mathrm{min}$ for $10 \mathrm{~min}$, dissociation $-60 \mu \mathrm{l} / \mathrm{min}$ of HBS for $7 \mathrm{~min}$, regeneration $-60 \mu \mathrm{l} / \mathrm{min}$ of $0.5 \mathrm{M}$ lactose in $0.1 \mathrm{M} \mathrm{HCl}$ for $10 \mathrm{~min}$, final HBS flow rate $-20 \mu \mathrm{l} / \mathrm{min}$ for $5 \mathrm{~min}$.
Kinetic parameters. The equilibrium dissociation constant $K_{\mathrm{D}}$ was calculated based on the binding data, using the BIAevaluation version 3 program (Biacore AB, Uppsala, Sweden).

\section{RESULTS AND DISCUSSION}

\section{Binding experiments}

We measured interaction of three lectins with human glycophorin A. Two lectins $-P$. velutina lectin (PVL) and T. vulgaris agglutinin (WGA) have similar specificity, i.e. they recognize two carbohydrate epitopes: GlcNAc and Neu5Ac residues. The third lectin, $S$. nigra (SNA-I), recognizes only one of those epitopes, i.e. Neu5Ac. Therefore, for desialylated glycophorin we could expect diminished binding with PVL and WGA, and close to zero binding with SNA-I.

\section{Psathyrella velutina lectin (PVL)}

The multi-ligand lectin from the fruiting body of $P$. velutina mushroom (PVL) is known to react best with terminal, non-reducing $\mathrm{N}$-acetylglucosamine and $\mathrm{N}$-acetylneuraminic acid (Neu5Ac) residues (Ueda et al., 1999a; 1999b). It was also found that the differential binding of oligosaccharides to PVL is due both to the number as well as the linkage of the non-reducing terminal carbohydrate residues, distinguishable by the lectin (Ueda et al., 1999a). For example, the sequence GlcNAc $\beta 1-2 \mathrm{Man} \alpha 1$... reacts with PVL more strongly than GlcNAc linked $\beta 1-4$ or $\beta 1-6$ to mannose (Endo et al., 1992). Accordingly, PVL has been applied to detect glycosylation abnormality in rheumatoid arthritis (RA) IgG (Tsuchiya et al., 1993). In IgG of RA patients there is a substantial lack of galactose residues at the non-reducing ends of Asn-297-linked N-glycans. The absence of these Gal residues exposes terminal, 
non-reducing GlcNAc residues which can be detected using appropriate reagent, e.g. a lectin.

During the pre-concentration procedure performed for PV lectin, the optimum $\mathrm{pH}$ at which the lectin gave the highest SPR response, was 5.4. After immobilizing the lectin onto the working channel the SPR baseline rose by $7340 \mathrm{RU}$. This is a relatively big change of the SPR signal which, according to the literature, may be a result of the known tendency of PVL to aggregate (Kochibe \& Matta, 1989). If this is the case then the layer of this lectin, created during immobilization, is not monomolecular but thicker, giving more carbohydrate-binding sites. Figure 1 presents an example sensorgram of the bind- from the SPR detector, which is considered as "binding". The binding of different glycophorin samples to PVL is presented in Fig. 2; this interaction was measured for both native and desialylated glycophorin samples. The binding of GPA to PV lectin was relatively strong (about $1200 \mathrm{RU}$ ); the glycophorin concentration in the analyzed solution was 0.083 $\mu \mathrm{M}(0.5 \mu \mathrm{g}$ protein $/ 200 \mu \mathrm{l}$ HBS $)$. As can be seen, the interaction of immobilized PVL with different native glycophorin samples is similar, regardless of the blood group of the erythrocytes which were the source of these glycophorin samples. Slightly lower binding was observed for GPA-A samples, as compared with GPA-B and GPA-O. All desialylated samples showed clearly lower binding, as com-

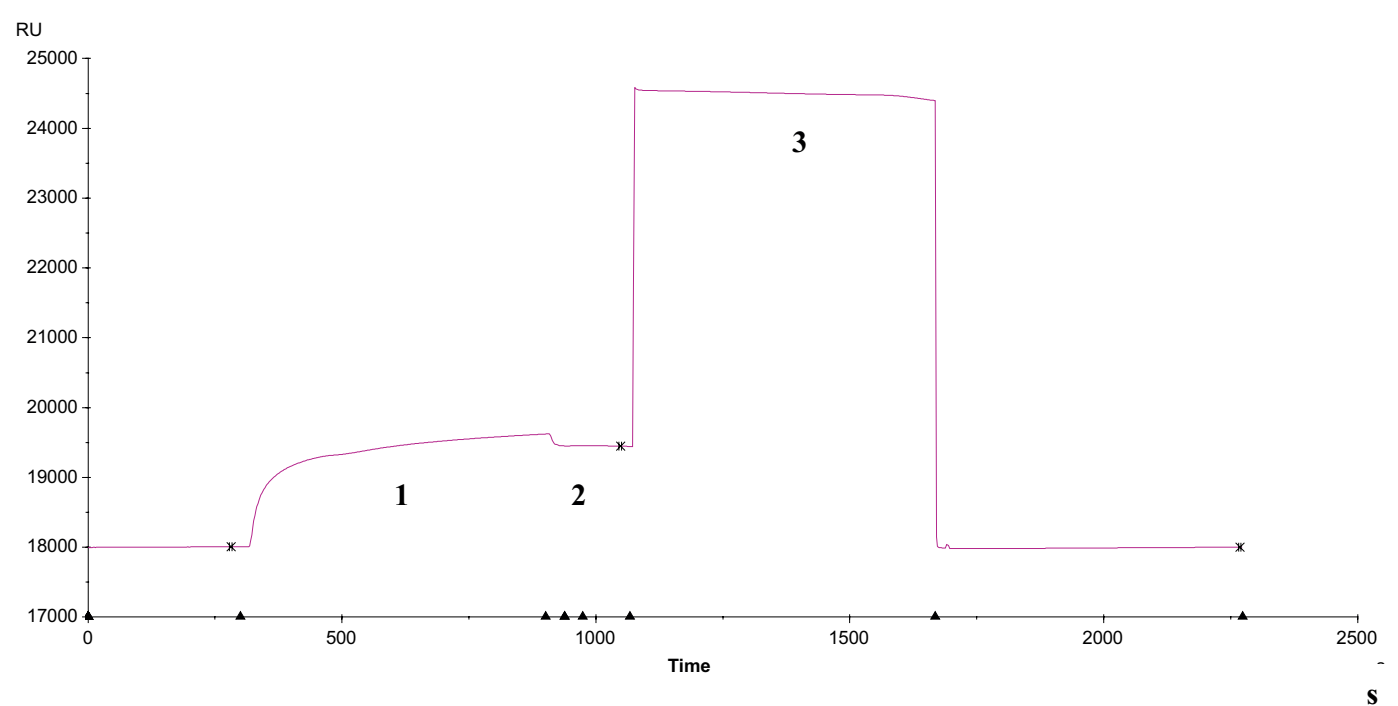

Figure 1. Example sensorgram showing the interaction between immobilized PVL lectin and human glycophorin.

GPA concentration was $0.5 \mu \mathrm{g}$ protein $/ 200 \mu \mathrm{l}$ of HBS buffer. The experiment consisted of the following steps: 1, association, $10 \mathrm{~min}$; 2, dissociation, $3 \mathrm{~min}$; 3, regeneration of the channel, $10 \mathrm{~min}$. Sensorgrams showing interaction of glycophorin samples with two other immobilized lectins, WGA and SNA-I, were entirely the same.

ing experiment obtained for human glycophorin as the analyte and immobilized PV lectin. The sensorgram exhibits three typical experimental time intervals: association, dissociation and regeneration of the working channel. The dissociation removes non-specifically bound molecules of the analyte; at the end of this time interval the instrument automatically measures the signal pared with the parent ones, Fig. 2-II. This agrees with the described specificity of PVL. Estimation of sialic acid in the desialylated samples revealed the presence of $2-4 \%$ sialic acid relative to protein content (not shown). Therefore, the residual presence of Neu5Ac in the desialylated samples together with the presence of bi-secting GlcNAc residues in $\mathrm{N}$-glycans explain the interaction of desia- 
lylated GPA samples with PVL. As shown in Fig. 2, purified samples of GPA-A, GPA-B and GPA-O exhibit very similar binding, which is lower than that of crude GPA preparations. It is probable, therefore, that the crude glycophorin preparations may include some amount of carbohydrate-containing impurities (e.g., glycolipids), interacting with PV lectin and making the SPR response of the sample slightly higher. One should also keep in mind that the glycophorin samples analyzed here originated from erythrocytes of individual persons and there always is a possibility that differences in the interaction of such individual glycophorin preparations with a lectin may reflect differences in glycosylation of these glycophorin samples, based on native microheterogeneity or the health status of the donor. The latter has been described, i.e. human glycophorin A was shown to be underglycosylated in CDG patients (Krotkiewska et al., 1999; Zdebska et $a l ., 2001)$.

Triticum vulgaris lectin (wheat-germ agglutinin, WGA)

This lectin is a dimer of two identical $18 \mathrm{kDa}$ subunits, each consisting of four homologous domains of 43 amino acids each. WGA specifically recognizes GlcNAc and Neu5Ac. The binding interactions, observed in crystal complexes with WGA (Wright \& Kellogg, 1996) are consistent with the presence ot two high affinity and two low affinity, thermodynamically independent sites per subunit, which means eight independent carbohydrate binding sites per one lectin molecule. From the results of affinity chromatography on WGA-agarose it may be suggested that there are different binding sites for GlcNAc and Neu5Ac in a WGA molecule (Gallagher et al., 1985).

During the pre-concentration procedure performed for WGA lectin the optimum $\mathrm{pH}$ of sodium acetate buffer was 4.5. This buffer was used, therefore, for the immobilization of the lectin in the working channel, which resulted in an SPR baseline rise by $4180 \mathrm{RU}$. The results of binding, measured for different GPA samples and immobilized WGA lectin, are shown in the Fig. 3. A characteristic feature of this interaction is clearly a weaker SPR response, compared with GPA-PVL interaction (Fig. 2). In the binding experiments with WGA, glycophorin samples had the concentration $0.83 \mu \mathrm{M}(2.5 \mu \mathrm{g}$ protein $/ 100 \mu \mathrm{l}$ HBS), which was 10 times more than in the experiments with PVL. For the native, crude glycophorin preparations the highest reaction was for GPA-A, and a little weaker for GPA-B and GPA-O. The corresponding desialylated preparations gave lower SPR responses, which were comparable to GPA binding to PVL (Fig. 2). Three purified, native preparations, one of each blood group, exhibited almost the same binding, around $170 \mathrm{RU}$. These
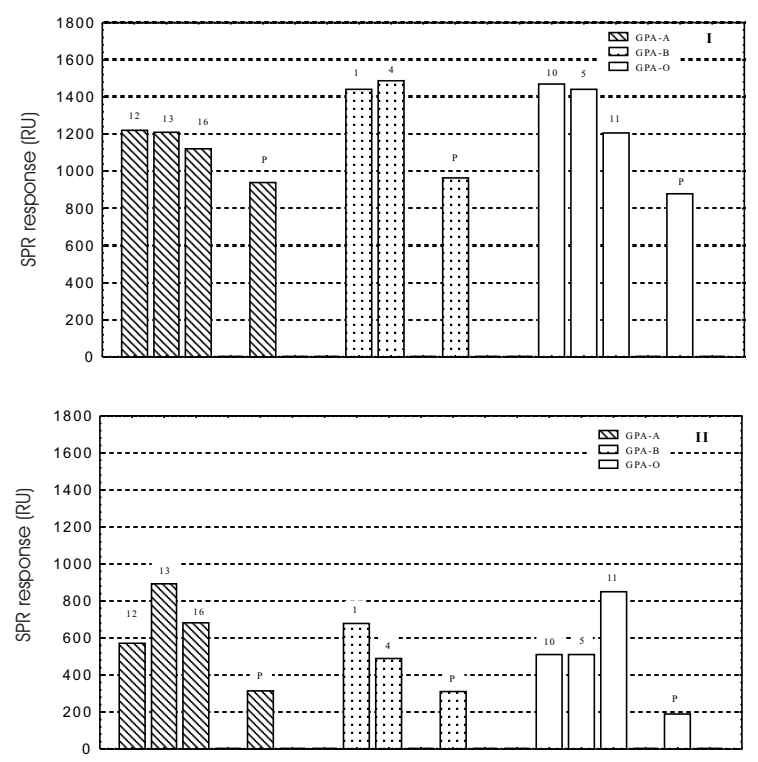

Figure 2. Results of GPA binding to immobilized PV lectin.

Glycophorin samples originated from individual subjects indicated by numbers over the bars. Concentration of all glycophorin samples, native and desialylated, was $0.5 \mu \mathrm{g}$ protein $/ 200 \mu \mathrm{l}$ of HBS buffer $(0.083 \mu \mathrm{M})$. The bars represent mean value of two measurements, which always differed by less than $5 \%$. In the control experiment the binding of BSA was negligible (less than 10\%). I, native glycophorin; II, desialylated glycophorin; $p$, purified sample. 
values were higher than for crude preparations, except for one GPA-A preparation. After desialylation these three samples showed lower reactivity, similar to desialylated crude glycophorin samples. Similarly to PVL, Fig. 2, desialylated glycophorin samples still reacted with WGA. According to the lectin specificity this binding probably resulted from the fact that WGA, like PVL, exhibits double specificity (GlcNAc and Neu5Ac) and desialylated GPA still contained sugar residues (i.e. the bisecting GlcNAc in N-glycans and residual sialic acid) recognizable by the lectin.

\section{Sambucus nigra lectin I (SNA-I)}

SNA-I lectin is isolated from a partially purified bark extract of elderberry tree; it exhibits specificity towards Neu5Ac $\alpha(2-6) \mathrm{Gal} /$ GalNAc sequence (Van Damme et al., 1996). Both types of receptors exist in human glycophorin, i.e. Neu5Ac $\alpha 2-6 \mathrm{Gal} \beta 1 . .$. exist as non-reducing termini in N-glycan and Neu5Ac $\alpha 2$ 6GalNAc sequence is present in most O-glycans. Therefore, human glycophorin can react with SNA-I.

During the pre-concentration procedure performed for SNA-I lectin the optimum $\mathrm{pH}$ of sodium acetate buffer was 4.0. This buffer was used, therefore, for the immobilization of the lectin onto the working channel; after the procedure was completed the SPR baseline rose by 2650 RU. During binding experiments the concentration of the glycophorin solutions was maintained at $0.83 \mu \mathrm{M}(2.5 \mu \mathrm{g}$ protein/100 $\mu \mathrm{l} \mathrm{HBS),} \mathrm{similarly} \mathrm{like} \mathrm{for} \mathrm{WGA.} \mathrm{The}$ results of binding are presented in Fig. 4. As can be seen, the magnitudes of the reactions are comparable for the samples from different blood groups; these bindings are a little lower than with WGA lectin (Fig. 3). For desialylated GPA samples the reaction is very low, in some cases close to zero. This is in agreement with the described specificity of SNA-I lectin: if the efficiency of the desialylation procedure is close to $100 \%$, the reaction of glycophorin with SNA-I drops close to zero.
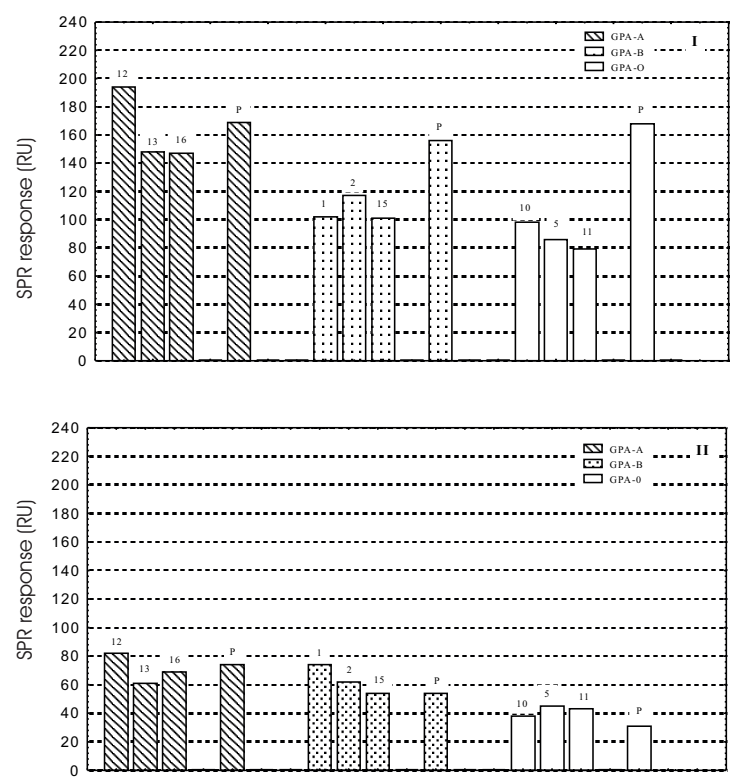

Figure 3. Results of GPA binding to immobilized WGA lectin.

Glycophorin samples originated from individual subjects. Concentration of all glycophorin samples, native and desialylated, was $2.5 \mu \mathrm{g}$ protein $/ 100 \mu \mathrm{l}$ of HBS buffer $(0.83 \mu \mathrm{M})$. For further explanations see legend to Fig. 2.

\section{Kinetic parameters}

We have calculated dissociation constants $K_{\mathrm{D}}$, which indicate the concentration of the analyte (lectin in this case) in the equilibrium state. If the $K_{\mathrm{D}}$ value is higher, the binding of the analyte to the immobilized ligand is weaker and vice versa. We performed this calculation by fitting each measurement data to a single site interaction model, which is a common procedure used in this type of experiments (Myszka et al., 1998). The results are summarized in Table 1. As can be seen, different $K_{\mathrm{D}}$ values were obtained for different lectins. For WGA the calculated $K_{\mathrm{D}}$ values were the highest. They exhibited a regular tendency, i.e. for the native GPA samples $K_{\mathrm{D}}$ values were lower than for the desialylated ones, for all three blood groups. The same tendency can be seen for the interaction of glycophorin with PVL, although in this case $K_{\mathrm{D}}$ values 
Table 1. Equilibrium dissociation constants $K_{\mathrm{D}}$, calculated for complexes of three types of GPA with different lectins

\begin{tabular}{|c|c|c|c|c|}
\hline \multicolumn{2}{|c|}{ Glycophorin } & WGA & PVL & SNA-I \\
\hline \multirow[t]{2}{*}{ GPA-A } & -native & $175 \mathrm{nM}$ & $4.3 \mathrm{nM}$ & $38 \mathrm{nM}$ \\
\hline & -desialylated & $300 \mathrm{nM}$ & $5.0 \mathrm{nM}$ & $1.6 \mathrm{nM}$ \\
\hline \multirow[t]{2}{*}{ GPA-B } & -native & $26 \mathrm{nM}$ & n.d. & 42 nM \\
\hline & -desialylated & $150 \mathrm{nM}$ & $8.4 \mathrm{nM}$ & $0.4 \mathrm{nM}$ \\
\hline \multirow{2}{*}{ GPA-O } & -native & $270 \mathrm{nM}$ & 3.9 n.M & $41 \mathrm{nM}$ \\
\hline & -desialylated & $475 \mathrm{nM}$ & $12.3 \mathrm{nM}$ & $2 \mathrm{nM}$ \\
\hline
\end{tabular}

n.d., not determined (in this case $K_{\mathrm{D}}$ could not be calculated).

were more than 10 times lower (in comparison with WGA). This result indicates that interaction of glycophorin with PVL is stronger and more specific than with WGA. A reciprocal tendency of $K_{\mathrm{D}}$ values was observed in the case of SNA-I lectin. Although the absolute binding of GPA was lower for desialylated samples, see Fig. 4 , the $K_{\mathrm{D}}$ values were very low (0.4-2 nM). To the latter result two explanations seem to be applicable: first - the binding of desialylated GPA to SNA-I is so weak that the equilibrium concentration of the analyte cannot be higher than about $2 \mathrm{nM}$; second - during desialylation of GPA the majority of the sugar epitopes recognizable by SNA-I lectin were lost, but some new epitopes appeared giving a strong binding of the lectin. We plan to explain this phenomenon in further experiments, using other GPA-binding lectins.

\section{CONCLUSIONS}

Based on the results obtained in this investigation one can conclude that the biosensor BIAcore, with SPR as a detection method, is a powerful method for investigating the interaction between lectins and glycoproteins. Measurement of the interaction between glycophorin and lectin may serve to detect even small changes in the carbohydrate moiety of this glycoprotein. The results showed that there is a small difference in binding between crude and purified glycophorin sam-
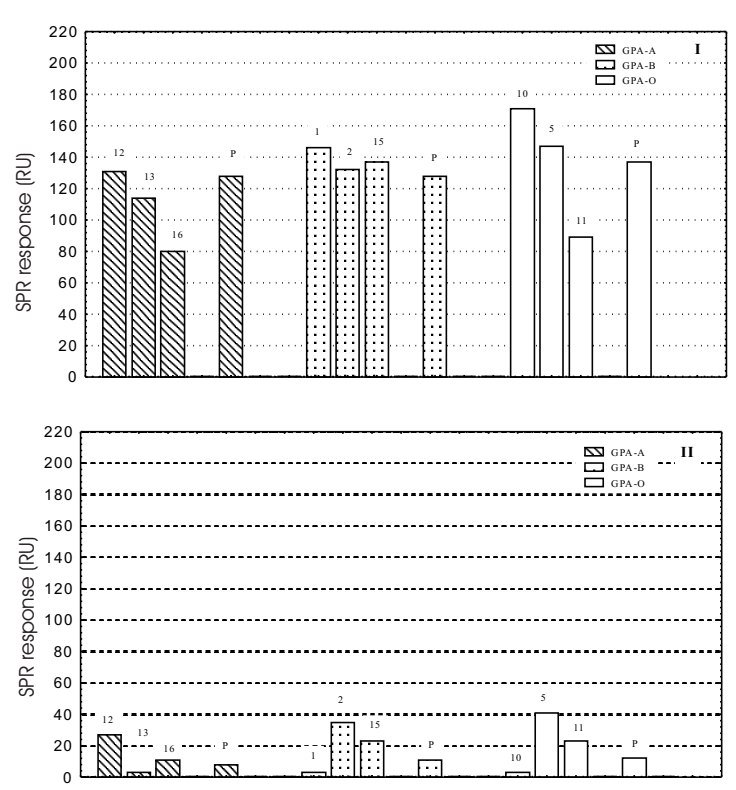

Figure 4. Results of GPA binding to immobilized SNA-I lectin.

Glycophorin samples originated from individual subjects. Concentration of all glycophorin samples, native and desialylated, was $2.5 \mu \mathrm{g}$ protein/100 $\mu \mathrm{l}$ of HBS buffer $(0.83 \mu \mathrm{M})$. For further explanations see legend to Fig. 2.

ples. It means that there is a possibility to analyze glycophorin preparations derived from small (e.g., $1 \mathrm{ml}$ ) blood samples, not suitable for standard purification. We plan to apply this finding to the analysis of GPA samples derived from patients with defined diseases. Finally, we noticed during the experiments that lectin immobilized in a single working channel could serve to perform over 200 binding measurements. 
The authors thank Professor E. Lisowska for valuable comments on the manuscript.

\section{R E F E R E N C E S}

Endo T, Ohbayashi H, Kanazawa K, Kochibe N, Kobata A. (1992) Carbohydrate binding specificity of immobilized Psathyrella velutina lectin. J Biol Chem.; 267: 707-13.

Gallagher JT, Morris A, Dexter TM. (1985) Identification of two binding sites for wheat-germ agglutinin on polylactosamine-type oligosaccharides. Biochem J.; 231: 115-22.

Hayashi K. (1975) A rapid determination of sodium dodecyl sulfate with methylene blue. Anal Biochem.; 67: 503-6.

Johnsson B, Löfås S, Lindquist G. (1991) Immobilization of proteins to a carboxymethyldextran-modified gold surface for biospecific interaction analysis in surface plasmon resonance sensors. Anal Biochem.; 198: 268-77.

Jourdian GW, Dean L, Roseman S. (1971) The sialic acids. A periodate-resorcinol method for the quantitative estimation of free sialic acids and their glycosides. J Biol Chem.; 246: $430-5$.

Kochibe N, Matta KL. (1989) Purification and properties of an $N$-acetylglucosamine-specific lectin from Psathyrella velutina mushroom. $J$ Biol Chem.; 264: 173-7.

Krotkiewski H, Lisowska E, Nilsson G, Grönberg G, Nilsson B. (1993) An improved approach to the analysis of the structure of small oligosaccharides of glycoproteins: application to the O-linked oligosaccharides from human glycophorin A. Carbohydr Res.; 239: 35-50.

Krotkiewski H, Duk M, Syper D, Lis H, Sharon N, Lisowska E. (1997) Blood group $\mathrm{MN}$-dependent difference in degree of galactosylation of O-glycans of glycophorin A is restricted to the GalNAc residues located on amino acid residues $2-4$ of the glycophorin polypeptide chain. FEBS Lett.; 406: $296-300$.
Krotkiewska B, Zwierz K, Krotkiewski H. (1999) The carbohydrate moiety of human glycophorin in CDG syndrome. Acta Biochim Polon.; 46: 371-6.

Lisowska E. (2001) Antigenic properties of human glycophorin - an update. Adv Exp Med Biol.; 491: 155-69.

Lisowska E, Duk M, Dahr W. (1980) Comparison of alkali-labile oligosaccharide chains of M. and $\mathrm{N}$ blood-group glycopeptides from human erythrocyte membrane. Carbohydr Res.; 79: 103-13.

Lisowska E, Messeter L, Duk M, Czerwiński M, Lundblad A. (1987) A monoclonal anti-glycophorin A antibody recognizing the blood group M. determinant: Studies on the subspecificity. Mol Immunol.; 24: 605-13.

Myszka DG, Jonsen MD, Graves BJ. (1998) Equilibrium analysis of high affinity interactions using BIACORE. Anal Biochem.; 265: 326-30.

Podbielska M, Krotkiewski H. (2000) Identification of blood group A and B antigens in human glycophorin. Arch Immunol Ther Exp.; 48: 211-21.

Quinn JG, O’Kennedy R, Smyth M, Moulds J, Frame T. (1997) Detection of blood group antigens utilising immobilised antibodies and surface plasmon resonance. J Immunol Methods.; 206: 87-96.

Rich RL, Myszka DG. (2000) Survey of the 1999 surface plasmon resonance biosensor literature. J Mol Recognit.; 13: 388-407.

Smith PK, Krohn RI, Hermanson GT, Mallia AK, Gartner FH, Provenzano MD, Fujimoto EK, Goeke NM, Olson BJ, Klenk DC. (1985) Measurement of protein using bicinchoninic acid. Anal Biochem.; 150: 76-85.

Stenberg E, Persson B, Roos H, Urbaniczky C. (1991) Quantitative determination of surface concentration of protein with surface plasmon resonance by using radiolabeled proteins. J Colloid Interface Sci.; 143: 513-26.

Tsuchiya N, Endo T, Matsuta K, Yoshinoya S, Takeuchi F, Nagano Y, Shiota M, Furukawa K, Kochibe N, Ito K, Kobata A. (1993) Detection of glycosylation abnormality in rheuma- 
toid IgG using $N$-acetylglucosamine-specific Psathyrella velutina lectin. J Immunol.; 151: 1137-46.

Ueda H, Kojima K, Saitoh T, Ogawa H. (1999a) Interaction of a lectin from Psathyrella velutina mushroom with $N$-acetylneuraminic acid. FEBS Lett.; 448: 75- 80.

Ueda H, Saitoh T, Kojima K, Ogawa H. (1999b) Multi-specificity of a Psathyrella velutina mushroom lectin: heparin/pectin binding occurs at a site different from the $\mathrm{N}$-acetylglucosamine/ $\mathrm{N}$-acetylneuraminic acid-specific site. J Biochem.; 126: 530-7.

Van Damme EJM, Barre A, Rouge P, Van Leuven F, Peumans WJ. (1996) The

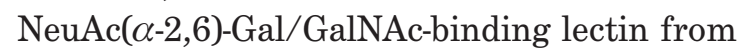
elderberry (Sambucus nigra) bark, a type-2 ri- bosome-inactivating protein with an unusual specificity and structure. Eur J Biochem.; 235: 128-37.

Wright CS, Kellogg GE. (1996) Differences in hydropathic properties of ligand binding at four independent sites in wheat germ agglutinin-oligosaccharide crystal complexes. Protein Sci.; 5: 1466-76.

Yoshima H, Furthmayr H, Kobata A. (1980) Structures of the asparagine-linked sugar chains of glycophorin A. J Biol Chem.; 255: 9713-8.

Zdebska E, Musielak M, Jaeken J, Kościelak J. (2001) Band 3 glycoprotein and glycophorin A from erythrocytes of children with congenital disorder of glycosylation type-Ia are underglycosylated. Proteomics.; 1: 269-74. 